\title{
Development of Teleological Explanations in Peruvian Quechua-Speaking and U.S. English-Speaking Preschoolers and Adults
}

\author{
Ingrid Sánchez Tapia, Susan A. Gelman, and \\ Michelle A. Hollander \\ University of Michigan
}

\author{
Bruce Mannheim \\ University of Michigan
}

\author{
Erika M. Manczak \\ Northwestern University
}

\author{
Carmen Escalante \\ Universidad Nacional San Antonio Abad del Cuzco
}

\begin{abstract}
Teleological reasoning involves the assumption that entities exist for a purpose (giraffes have long necks for reaching leaves). This study examines how teleological reasoning relates to cultural context, by studying teleological reasoning in 61 Quechua-speaking Peruvian preschoolers $\left(M_{\mathrm{age}}=5.3\right.$ years $)$ and adults in an indigenous community, compared to 72 English-speaking U.S. preschoolers $\left(M_{\text {age }}=4.9\right.$ years $)$ and university students. Data were responses to open-ended "why" questions ("Why is that mountain tall?"). Teleological explanations about nonliving natural kinds were more frequent for children than adults, and for Quechua than U.S. participants. However, changes with age were importantly distinct from differences corresponding to cultural variation. Developmental and cultural differences in teleological explanations may reflect causal analysis of the features under consideration.
\end{abstract}

From an early age, children treat entities in their world as existing for a purpose (e.g., clocks are for telling time; a giraffe's neck is for reaching leaves). This form of thought, known as teleological reasoning, is central to how people construct explanatory theories of the world. Teleological reasoning is not only a key component of intuitive theory building (Kelemen, 1999a), but also has important implications for scientific literacy, as it can interfere with understanding mechanistic causal processes (Gregory, 2009; Kelemen, 2012).

An unresolved debate concerns the domains to which teleological explanations are applied. Two dominant positions have been set forth: selective teleology (ST) and promiscuous teleology (PT; Kelemen, 1999a). According to ST, children and adults alike have a "design stance" that is limited to artifacts and biological entities, though the nature of that stance differs in the two domains (Greif, Kemler Nelson, Keil, \& Gutierrez, 2006; Keil, 1995). Arti-

This research was supported by NICHD Grant HD-36043 to Susan A. Gelman. We are grateful to the children, parents, and schools that participated in the studies, including Poques Preschool. We thank Beth Anastasia, Martin Castillo, Natalie Davidson, Laura Distel, Josh Shapero, and Alex Was for their research assistance.

Correspondence concerning this article should be addressed to Susan Gelman, 530 Church St., Department of Psychology, University of Michigan, Ann Arbor, MI 48109-1043. Electronic mail may be sent to gelman@umich.edu. facts and their properties function to serve humans; for example, a cup is designed for a person to drink from. In contrast, properties of biological entities function to serve the animal or plant itself; for example, a turtle's shell protects it from predators. ST is variously proposed to be a component of children's innate biological module, and so constrained to exclude certain kinds of entities (whole animals or plants, or nonliving natural kinds [NLNKs]; Atran, 1995), or the outcome of an autonomous mode of construal that is favored for the biological domain (Greif et al., 2006; Keil, 1995). Keil (1995) further proposes that domains differ in their underlying causal principles, and that these principles are responsible for different modes of explanation. In contrast, the PT model proposes that there is a broad human tendency to apply teleological explanations across domains, including not just artifacts and parts of living kinds, but also NLNKs (mountains are tall for people to climb) and whole animals (an animal is for walking around; Kelemen, 1999b). Eventually children learn to restrict this tendency, but even adults revert to PT under speeded conditions (Kelemen \& Rosset, 2009) or

(C) 2016 The Authors

Child Development (C) 2016 Society for Research in Child Development, Inc. All rights reserved. 0009-3920/2016/8703-0011

DOI: $10.1111 /$ cdev.12497 
cognitive decline (Lombrozo, Kelemen, \& Zaitchik, 2007).

Although there is much evidence from the United States and Britain in middle-class, urban, or suburban communities, primarily supportive of PT, little is known about teleological reasoning in other contexts. This is a serious limitation. Industrialized communities in which children receive extensive formal education and exposure to Western science are unusual the world over (Henrich, Heine, \& Norenzayan, 2010; Mazzocchi, 2006). It is thus an open question whether the patterns obtained in the United States and Britain would extend to other contexts. Moreover, examining teleological reasoning in other cultural groups permits one to investigate why teleological explanations are less frequent in adults than children.

For these reasons, Casler and Kelemen (2008) studied Romanian Romani adults with varied levels of schooling. Interestingly, those with less formal schooling applied teleological reasoning to NLNKs, much as did U.S. children in earlier work. Yet more investigations of this nature are needed. For example, people in more rural and isolated communities have rich ecological knowledge of the natural world (e.g., different types of plants for feeding animals, cooking, etc.), which could increase attention to purpose or function.

Finally, past work has rarely examined the content of participants' teleological explanations. On one hand, teleological purpose may be construed in an explicitly artifact-like manner, as designed for human purpose (i.e., anthropocentrism). Although PT does not necessarily imply anthropocentrism, some examples in the literature are of this sort (e.g., a mountain is "to climb"; Kelemen, 1999b). On the other hand, in contrast to the assumption of PT that teleological reasoning indexes intentional design, ojalehto, Waxman, and Medin (2013) propose that "teleological thinking about nature reflects relational reasoning about perspectival relations among living things and their environments" (p. 166). For example, "Trees provide a home for birds" indexes a relation between two elements in nature (birds and trees) and a particular perspective (i.e., the bird's point of view). They call this a "relational-deictic" framework, which we dub "RDT" (relational-deictic teleology).

We address these issues by studying preschool children and adults in two cultural groups: English speakers in a middle-class university community in the United States, and Quechua speakers in a rural community in the Peruvian Andes. Our goal is to examine how teleological reasoning relates to broad features of cultural context, rather than focusing on a single dimension of cultural variation (e.g., schooling, as in Casler \& Kelemen, 2008; religion, as in Diesendruck \& Haber, 2009). We compared a sample that is typical of prior research (intensively schooled, relatively secular community within the United States) with one that has never before been studied with respect to these issues (indigenous, rural, Quechua-speaking community).

The Quechua-speaking context was chosen because it is an indigenous community that differs in numerous respects from populations that have been studied previously, including rural/agricultural, fewer years of formal schooling, more religious, and highly attentive to relational patterns in nature (Orlove, Chiang, \& Cane, 2002). Adults in this community have typically no more than 5 years of school, yet both children and adults have intensive experience with the natural world via farming and raising animals.

Additionally, Quechua (which is the primary language spoken in this community) expresses teleological function or purpose with a grammatical marker (-paq), which appears on nouns and nominalized verbs (Cusihuamán Gutiérrez, 1976). The meanings of -paq that were of greatest interest to us are use (Ima-paq-taq kay qurari allin?/"What is this qura herb for?") and goal or persistent objective (Chakrachikunay-paq-mi aqhashani/"I'm making maize beer in order to work my field."). Other uses are similar to those of English "for" (e.g., recipient of an object: Taytayki-paq-mi chay ahaqa kashan/ "That maize beer is for your father"), though content and context typically clarify the intended meaning (Cusihuamán Gutiérrez, 1976). Because -paq is grammaticized, it provides a readily accessible means of expressing teleology. Furthermore, parents use -paq highly frequently when speaking about the purpose of whole items (especially artifacts) with their 5-year-old children (Gelman, Mannheim, Escalante, \& Sánchez Tapia, 2015). This raises the possibility that teleological reasoning would be particularly salient in this community.

We examined three questions regarding: domain specificity, overall frequency, and content of teleological explanations. (a) Domain specificity: Do Quechua speakers provide more teleological explanations for properties of NLNKs? Kelemen's intentional design theory (Kelemen \& DiYanni, 2005) would make this prediction, as it proposes that PT is a domain-general tendency reflecting an intentional stance that is suppressed with formal instruction in Western scientific theories. In contrast, Keil (1995) and Greif et al. (2006) would predict domain-specific patterns in both children and 
adults in both cultural communities, with teleological explanations restricted to artifacts and adaptive parts of animals. (b) Overall frequency: Do Quechua participants overall appeal to teleological explanations more than U.S. participants, due to their limited exposure to Western science (which conveys mechanistic explanations that may supplant teleology; Casler \& Kelemen, 2005) and greater emphasis on religion (which promotes an intentional stance; Diesendruck \& Haber, 2009)? (c) Content: How often does teleological reasoning reflect a tendency to treat the natural world as intentionally created for human purposes? And in contrast, how often does it reflect a sensitivity to relatedness among elements of nature and contrasting perspectives? Among the Quechua, farmers understand rain as a necessity for agriculture, and NLNKs such as stars and clouds are consulted to organize agricultural activities and life events, and to predict climatic conditions. The observation that the brightness and size of certain stars can be used to forecast the timing for planting potato plants is common among Quechua speakers in Peru and Bolivia, reflecting sensitivity to patterns that non-Quechua scientists discovered only recently (Orlove et al., 2002).

We posed a series of open-ended "why" questions (e.g., "Why is that star twinkly?") to elicit explanations that could be coded as teleological (e.g., "Our god in heaven made the stars in order to shine on us people"), causal (e.g., "They are balls of fire so they appear shiny"), and/or nonexplanatory (e.g., "It's actually Venus"). Kelemen, Callanan, Casler, and Pérez-Granados (2005) distinguished "teleological" explanations (focused on purpose or function) from "causal" explanations (focused on prior circumstances, including mechanisms, preconditions, and precipitating factors). This approach contrasts with much prior work, which asked participants to select the "best" explanation from a set of experimenter-supplied options (Keil, 1995; Kelemen, 1999c). Although experimenter-provided choices maintain strict control over explanatory responses, participant-generated choices yield insights into everyday reasoning. Similar methods have been used to examine teleological reasoning in children's questions about unfamiliar items (Greif et al., 2006) or responses to explicit prompts for teleological explanations ("What's the X for?"; Kelemen, 1999b). To our knowledge, however, no prior research has examined teleological explanations in response to experimenter-generated "why" questions.

Questions spanned three domains (NLNKs, animals, and artifacts) and two property types (static properties and behaviors) to assess the breadth of teleological reasoning. Much prior work on teleological explanations focused on static features, such as an animal's color or the texture of rocks. We hypothesized that teleological explanations might be more frequently employed for static features than behaviors. For U.S. adults, features that result from intentional design are privileged for teleological explanation (Lombrozo \& Carey, 2006), and static features such as functional parts may overtly reflect an item's design, with affordances that even young children understand (e.g., the handle of a hammer is for grasping; the webbed feet of an otter are for swimming; Kelemen, 1999a; McCarrell \& Callanan, 1995). In contrast, actions may reflect design more indirectly (e.g., people design the gears of a clock, but they do not design a clock to tick; rather, ticking is an indirect consequence of the design).

Finally, we varied whether wording referred to categories, using generic language (e.g., "Why do dogs bark?"), or referred to individuals, using specific language (e.g., "Why is that dog barking?"). Four- and 5-year-old children provide relatively more teleological explanations in the context of generic language (e.g., snakes have holes in their teeth, "So they can swallow things") than specific language (e.g., a particular snake has holes in his teeth "Maybe because a bug came in its room, and it bited [sic] his teeth"; Cimpian \& Markman, 2009). By including both generic and specific wording, we can gauge how flexibly participants shift their use of teleological explanations as a function of the linguistic context.

\section{Method \\ Participants}

In Peru, participants were 27 adults (16 women, 11 men) and 34 preschoolers (20 boys, 14 girls; $M_{\text {age }}=5.31$ years, age range $=4.00-6.03$ ). Data were collected in August 2011. Six additional adults were tested but not included because of equipment failure or poor recording quality. The participants lived in a rural community of Quechua speakers in the Peruvian Andes. Adults received \$10 (U.S.) as a token of appreciation. The children attended the only preschool center in the community and were tested there or in their homes. Each child received a picture book and a t-shirt for participating. All participants were native speakers of Quechua. Forty-eight percent of adults were bilingual (Quechua/Spanish), on average learning Spanish at 
10 years of age (range $=5-17$ years). The rest spoke only Quechua. More details regarding the Quechua sample are provided in Appendix S1.

In the United States, participants were 32 college students (24 men, 8 women) and 40 preschoolers (24 boys, 16 girls; $M_{\text {age }}=4.91$ years, age range $=4.05-5.47$ ). Children were tested March through October 2009, and adults in November 2008. Two additional children were tested but not included, one for not providing a minimum number of responses and one for equipment failure. The college students were enrolled in an introductory psychology course at a large university in the Midwestern United States. Their participation fulfilled class requirements. The college students were 56\% White, 19\% African American, 19\% Asian or Asian American, and 6\% Other. The children attended local preschool centers or were tested in an on-campus laboratory. They were from communities in and around a small university town in the Midwestern United States; most participants were White and middle class. All participants were native speakers of English.

\section{Materials}

Items included eight NLNKs, eight animals, and eight artifacts, each accompanied by a color photograph displaying the target property (Table 1). In each domain, half the features were static (e.g., "Why are rocks pointy?") and half were actions (e.g., "Why do balls roll?"). To ensure that the items were familiar and culturally appropriate, there were slight differences across the two testing sites (e.g., "Why do giraffes have long necks?" [United States] vs. "Why do llamas have long necks?" [Peru]). Half the participants received questions in generic form, as in the examples above ("Why are rocks pointy?"); half received questions in specific form ("Why is that rock pointy?"). The study materials were initially written in English and Spanish and then translated into Quechua by two fluent speakers of Quechua, one of whom is a native Spanish speaker and the other of whom is a native English speaker and fully fluent in Spanish, upon consultation with a native Quechua speaker. The study materials were back-translated.

Table 1

Items Presented by Domain and Property (Generic Wording)

\begin{tabular}{|c|c|c|c|}
\hline Domain & Property type & Items & Quechua translations \\
\hline \multirow[t]{8}{*}{ NLNK } & \multirow[t]{4}{*}{ Action } & Why do clouds float in the sky? & ¿imaraykun phuyukuna hanaqpachapi purin? \\
\hline & & Why do stones sink in the water? & ¿imaraykun rumikuna unupi chinkan? \\
\hline & & Why do volcanoes shoot out lava [rivers flow]? & ¿imaraykun mayukuna sunchun? \\
\hline & & Why does ice melt? & ¿imaraykun rit'i ch'ullun? \\
\hline & \multirow[t]{4}{*}{ Static } & Why are rocks pointy? & ¿imaraykun qaqakuna suyt’u kanku? \\
\hline & & Why are mountains tall? & ¿imaraykun urqukuna hatun kankaray kanku? \\
\hline & & Why are stars twinkly? & ¿imanaqtinmi ch’askakuna lliphipinku? \\
\hline & & Why is gold shiny? & ¿imanaqtinmi quri k'anchan? \\
\hline \multirow[t]{8}{*}{ Animal } & \multirow[t]{4}{*}{ Action } & Why do koalas [monkeys] climb trees? & ¿imanaqtinmi kusillukuna llakita lluqhan? \\
\hline & & Why do dogs bark? & ¿imanaqtinmi allqukuna kanikun? \\
\hline & & Why do zebras [pumas] run? & ¿imanaqtinmi pumakuna phawan? \\
\hline & & Why do frogs catch flies? & ¿imanaqtinmi k'ayrakuna ch'uspikunata hap'inku? \\
\hline & \multirow[t]{4}{*}{ Static } & Why do pigeons have wings? & ¿imanaqtinmi urpikuna pharpayuq kanku? \\
\hline & & Why do giraffes [llamas] have long necks? & ¿imanaqtinmi llamakuna suni kunkayuq? \\
\hline & & Why do turtles have shells? & ¿imanaqtinmi tortugakuna runqhi qarayuq? \\
\hline & & Why do monkeys [cats] have tails? & ¿imanaqtinmi michikuna chupayuq kanku? \\
\hline \multirow[t]{8}{*}{ Artifact } & \multirow[t]{4}{*}{ Action } & Why do balls roll? & ¿imanaqtinmi pelota suchun? \\
\hline & & Why do clocks tick? & ¿imanaqtinmi relojkuna "tic tac" ninku? \\
\hline & & Why do telephones ring? & ¿imanaqtinmi telefonokuna wahamun? \\
\hline & & Why do boats float? & ¿imanaqtinmi barcokuna tuytun? \\
\hline & \multirow[t]{4}{*}{ Static } & Why do cars have wheels? & ¿imanaqtinmi automobilkuna ruedayuq kanku? \\
\hline & & Why do chairs have legs? & ¿imaraykun tiyanakuna chakikunayuq? \\
\hline & & Why do microwaves [radios] have buttons? & ¿imaraykun radiyukuna butunniyuq? \\
\hline & & Why do coats have pockets? & ¿imaraykun abrigokuna bolsilluyuq? \\
\hline
\end{tabular}

Note Where the U.S. and Quechua items differed, the Quechua version is in brackets. NLNK = nonliving natural kinds. 


\section{Procedure}

Peruvian Quechua participants and U.S. children were tested individually in their native language (English for U.S. participants; Quechua for Peruvian Quechua participants) by a female experimenter. The Peruvian interviewer was a Quechua-speaking out-group member with close ritual ties to members of the community (compadrazgo). She had built relationships with adult women and some elderly members of the community over a period of 10 15 years and was well known and trusted among them. In the United States, children were tested in a quiet room; in Peru, participants were tested in the schoolyard or the participant's home. Participants were told that they would be shown some pictures and asked if they could help out by answering questions about them. In the United States, children were additionally told that the questions came from a child.

For each of 24 questions, the experimenter first laid out a laminated photo of the target item (e.g., a turtle), then provided a framing statement (e.g., "I'm going to ask you a question about turtles"), followed by the question itself (e.g., "Why do turtles have shells?"). No feedback was provided, aside from simple nondirective responses ("OK," "good"). When children gave a nonresponse, they were encouraged to give their best guess. The order of items within a block was randomly determined, separately for each participant. Responses were audio recorded.

The U.S. adults were tested individually or in small groups in a quiet room, with a paper-and-pencil task (in English; but see Appendix S2 for a replication study using face-to-face interviews). Participants were asked each question separately and told not to go back and change their responses once a question was answered. For each item, participants saw a photo of the target item and the target question. This method provides continuity with prior studies of teleological reasoning in U.S. adults (Kelemen \& Rosset, 2009; Lombrozo \& Carey, 2006).

\section{Transcription/Translation and Coding}

For the U.S. participants, responses were transcribed verbatim. For the Quechua participants, the child responses were directly translated into Spanish from the recordings, by the researcher who conducted the Quechua interviews. The Quechua adult responses were transcribed and translated into Spanish and English. Due to limited availability of Quechua transcribers and translators, our highest priority was obtaining transcriptions for the adults, whose responses were longer, more conceptually sophisticated, and more grammatically complex. Spanish translations were also translated into English by a bilingual Spanish-English speaker who discussed all questions with the Quechua-speaking translators during the translation process. We had a multilingual research team (Quechua, Spanish, and/or English), and thus it was an advantage to have all three languages when possible. The order of translation was from Quechua to Spanish to English, because most of the Quechua translators knew Spanish but not English. Translations of the Quechua were conducted by three speakers bilingual in Quechua and Spanish, one of whom also is a native English speaker. These were not back-translated. Coding of Quechua responses was conducted from the English translations. For the adult responses, Quechua and Spanish transcriptions were also consulted. The primary coder was a native speaker of Spanish and fluent in English. When questions arose, the coders consulted a Quechua speaker.

Responses were coded for explanatory type: teleological (teleology or consequence), causal (prior cause), or nonexplanatory (Kelemen et al., 2005). Nonexplanatory responses were coded only if a teleological or causal response was not offered for the same item. We coded teleological responses for content as anthropocentric, ecorelational excluding humans ("ecorelational" for short), or neither. Anthropocentric indicated that the purpose was specifically for a human (e.g., "for people to walk there"); ecorelational expressed a link between the item and a nonhuman natural element, either living or nonliving (e.g., a river flows "in order for bugs to live, and in order for fish to live, in order for trout to live"); all others were neither (e.g., a pigeon has wings "to fly"). Humans are considered part of ecosystems within Western science. However, in order not to make assumptions about the role of humans within the Quechua ontology, the present coding of ecological relatedness excludes humans and yields a conservative estimate. Ecorelational teleological content was coded for NLNKs and animals only, as artifacts are not part of the natural ecosystem. An overview of the coding scheme, sample teleological responses, and all coding of causal ("prior cause") responses are provided in Appendices S3-S7.

For the coding of explanatory type in the U.S. data, one person coded all responses and a second coded $96 \%$ of responses. Interrater agreements were $85 \%$ (prior cause), 90\% (teleology), and 90\% (nonexplanatory); Cohen's kappas were .69 (prior cause), .80 (teleology), and .53 (nonexplanatory). For 
coding of explanatory type in the Quechua data, two people coded all responses. Interrater agreements were $85 \%$ (prior cause), 92\% (teleology), and $88 \%$ (nonexplanatory); Cohen's kappas were .69 (prior cause), .83 (teleology), and .54 (nonexplanatory). Two coders coded all files for teleological content, yielding agreements of $97 \%$ (United States) and 94\% (Quechua), and kappas of .93 (United States) and .86 (Quechua). With the exception of "nonexplanatory" responses (which were not analyzed further), all the kappas were either "substantial" (.61-.80) or "near perfect" (.81 and above), according to Landis and Koch (1977).

\section{Results}

The results are reported in four sections: a preliminary overview, then one section for each of the three domains (NLNKs, animals, and artifacts).

\section{Preliminary Overview}

Missing or unintelligible data accounted for less than $5 \%$ of the responses within each group. Most of the remaining responses were either teleological or causal (81\% for Quechua preschoolers, 89\% for U.S. preschoolers, 94\% for Quechua adults, and $96 \%$ for U.S. adults), and all but two participants provided both teleological and causal explanations at some point throughout the task. Given that nonexplanatory responses were rare $(11 \%$ of the data overall), that trials rarely included both a teleological and a causal explanation ( $4 \%$ of trials), and that children's teleological and causal responses were inversely correlated ( -.68 for United States, -.72 for Peru; $p s<.001$ ), we focus primarily on the teleological responses.

Initial analyses revealed a significant four-way interaction involving cultural context, age group, domain, and property type, $p=.034$; thus, the primary analyses are separated by domain. Additionally, we found substantially similar results for generic and specific wording: Generic wording yielded more teleological responses than specific wording, but only for the U.S. participants considering animal action items, $p=.001$. No other wording comparisons were significant. We therefore collapsed over this factor in all analyses reported henceforth. Finally, Quechua participants were more religious than this sample of U.S. participants, as confirmed by the percentage of participants who mentioned God at least once: $5 \%$ of U.S. children, $6 \%$ of U.S. adults, $18 \%$ of Quechua children, and
$67 \%$ of Quechua adults. Item analyses and individual response patterns are presented in Appendices S8 and S9.

\section{Nonliving Natural Kinds}

NLNKs provide the most direct test of PT and ST. Is there a tendency among U.S. and Quechua children and Quechua adults to provide more teleological explanations for properties of NLNKs (PT), or do we find similar, domain-specific patterns in both cultural communities and at both ages (ST)? Each participant's teleological responses were summed, separately for each property, with scores ranging from 0 to 4 (Figure 1a). We conducted a three-way analysis of variance (ANOVA) involving cultural context (Quechua, U.S.), age group (children, adults), and property type (static, action). As predicted by PT, we found higher rates of teleological explanations for children than adults, $F(1$, 129) $=21.04, p<.001, \eta_{\mathrm{p}}^{2}=.14$, and higher rates of teleological explanations among Quechua than U.S. participants, $F(1,129)=19.34, p<.001, \eta_{p}^{2}=.13$.

Teleological explanations were also significantly higher for static features than for actions, $F(1$, $129)=16.24, p<.001, \eta_{\mathrm{p}}^{2}=.11$, but this property difference interacted with both country, $F(1$, $129)=4.45, p=.037, \eta_{p}^{2}=.03$, and age group, $F(1$, $129)=7.84, p=.006, \eta_{p}^{2}=.06$, reflecting greater effects among the U.S. children, Quechua children, and Quechua adults. Nonetheless, effects of cultural context (more teleology among Quechua than U.S. participants) and age group (more teleology among children than adults) held up for both static and action properties, $p s \leq .036$, Bonferroni's correction.

We also classified participants into whether or not they ever produced a teleological explanation for any of the eight NLNK items. The majority of U.S. children (75\%), Quechua children $(74 \%)$, and Quechua adults (74\%) did produce at least one teleological explanation for NLNKs; by contrast, only $28 \%$ of U.S. adults ever did so.

How often does teleological reasoning about NLNKs reflect sensitivity to relatedness among elements of nature, as predicted by RDT? How often does it reflect a tendency to adopt an anthropocentric perspective? Here, the data reveal both developmental and cultural effects (Table 2). Anthropocentric responses were not uncommon, especially among children, $F(1,84)=9.67, p=.003, \eta_{p}^{2}=.11$. Yet participants also expressed ecorelational teleological functions, especially among Quechua participants, $F(1,129)=8.04, p=.005, \eta_{p}^{2}=.06$. For 
(a)
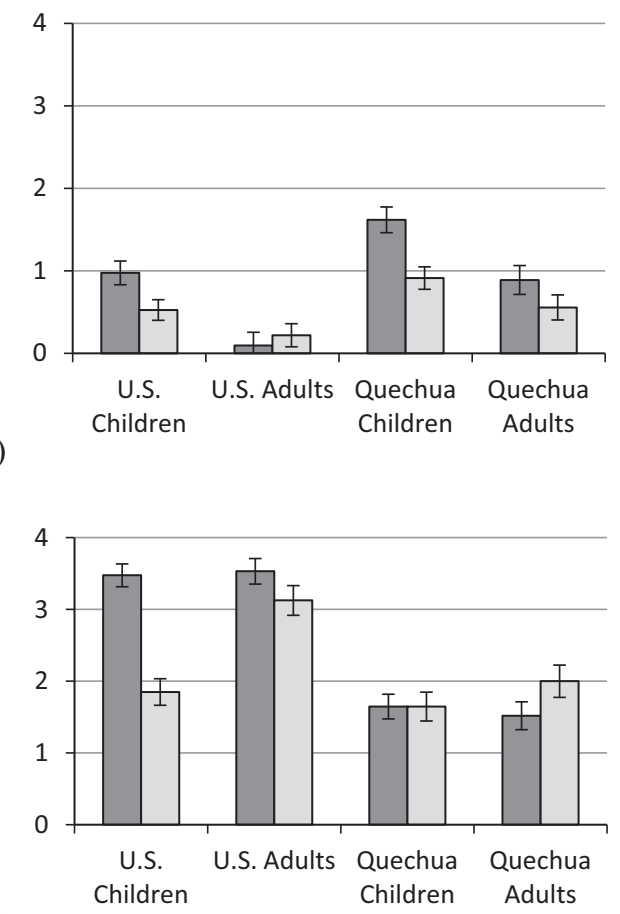

(b)

$\square$ Static

$\square$ Action $\square$ Action

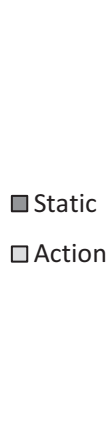

(c)

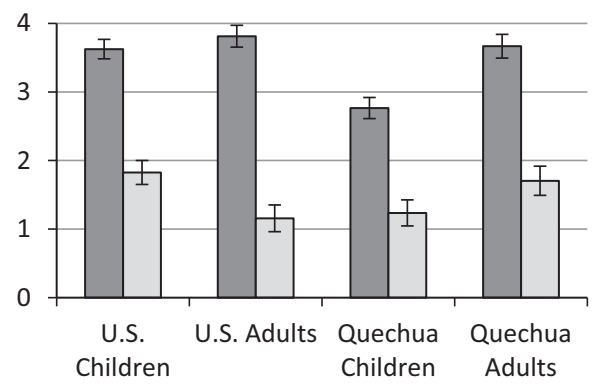

$\square$ Static

$\square$ Action

Figure 1. Mean number of teleological explanations as a function of age group, country, and property type. (a) Nonliving natural kinds, (b) animals, (c) artifacts. Scores could range from 0 to 4 .

example, one Quechua child said that rivers flow, "for fish to move inside the water." Furthermore, if one considers humans as part of the ecology, thus collapsing over "relational" and "anthropocentric" codes, all groups except the U.S. adults provided relational explanations on most teleological trials.

\section{Summary}

These findings at first appear consistent with the PT position that schooling and instruction in Western science suppress an otherwise promiscuous application of teleological explanations for NLNKs, with lowest rates of teleological explanations for U.S.
Table 2

Mean Percentage of Teleological Responses Coded as Ecorelational (Excluding Humans), Anthropocentric (Alternatively, Relational With Humans), or Neither

\begin{tabular}{lccc}
\hline & $\begin{array}{c}\text { Ecorelational } \\
\text { (excluding } \\
\text { humans) }\end{array}$ & $\begin{array}{c}\text { Anthropocentric } \\
\text { (relational } \\
\text { with humans) }\end{array}$ & Neither \\
\hline NLNKs & $18 \%$ & $58 \%$ & $24 \%$ \\
U.S. children & $11 \%$ & $22 \%$ & $67 \%$ \\
U.S. adults & $28 \%$ & $47 \%$ & $25 \%$ \\
Quechua children & $41 \%$ & $28 \%$ & $26 \%$ \\
Quechua adults & & & \\
Animals & $58 \%$ & $3 \%$ & $37 \%$ \\
U.S. children & $80 \%$ & $0 \%$ & $20 \%$ \\
U.S. adults & $45 \%$ & $9 \%$ & $46 \%$ \\
Quechua children & $68 \%$ & $6 \%$ & $26 \%$ \\
Quechua adults & - & $63 \%$ & $37 \%$ \\
Artifacts & - & $59 \%$ & $41 \%$ \\
U.S. children & - & $60 \%$ & $40 \%$ \\
U.S. adults & - & $70 \%$ & $30 \%$ \\
Quechua children & & & \\
Quechua adults & & & \\
\hline
\end{tabular}

Note. The ecorelational code was applied to the NLNK and animal domains only. NLNK = nonliving natural kinds.

adults and higher rates among U.S. children, Quechua children, and Quechua adults. Importantly, however, this is not an interaction, but rather two main effects (higher rates among children than adults, and higher rates among Quechua than U.S. participants), indicating that age and culture have independent influences. The underlying reasoning that participants provide, as reflected in the analyses of teleological content, also differs for the age effect (children are more anthropocentric than adults) versus the cultural effect (Quechua participants are more relational than U.S. participants). Altogether, for NLNKs, variation in teleological reasoning associated with age is not equivalent to variation in teleological reasoning associated with culture.

\section{Animals}

ST would predict high rates for all groups in the animal domain, for which teleological reasoning is argued to be privileged, and PT would also predict high rates, reflecting "broad purpose-based reasoning" (Casler \& Kelemen, 2008, p. 357). However, the animal domain provides an opportunity to test whether the groups differ in overall frequency of teleological reasoning. For example, Quechua children and adults may supply more teleological responses, given their greater religiosity 
and less experience with Western scientific instruction and schooling more generally. The mean numbers of teleological responses are displayed in Figure $1 \mathrm{~b}$. As with NLNKs, we conducted a 2 (age group) $\times 2$ (country) $\times 2$ (property) repeated measures ANOVA.

Counter to our predictions, teleological explanations for animals were higher among adults than children, $F(1,129)=5.71, p=.018, \eta_{p}^{2}=.04$, and among U.S. than Quechua participants, $F(1,129)=63.00$, $p<.001, \eta_{p}^{2}=.33$. Each of these main effects also interacted with property. Teleological explanations were more frequent for static than action animal properties, $F(1,129)=15.05, p<.001, \eta_{\mathrm{p}}^{2}=.10$, but this effect was carried exclusively by U.S. preschoolers, resulting in an Age Group $\times$ Property interaction, $F(1,129)=18.11, p<.001, \eta_{\mathrm{p}}^{2}=.12$, and a Country $\times$ Property interaction, $F(1,129)=39.55, p<.001$, $\eta_{\mathrm{p}}^{2}=.24$. Importantly, higher rates of teleology responses for animals among the U.S. than the Quechua participants held up for both property types, $p s=.004$, Bonferroni's correction.

How often does teleological reasoning reflect a tendency to treat animals from an anthropocentric perspective and/or sensitivity to relatedness among elements of nature? Most teleological explanations for animals were ecorelational; anthropocentric responses were extremely rare (see Table 2). A univariate ANOVA conducted on the proportion of teleological responses coded as relational indicated that adults were more relational than children, $F(1$, $129)=21.27, p<.001 \eta_{p}^{2}=.14$, and U.S. participants were more relational than Quechua participants, $F(1,129)=6.72, \quad p=.011, \quad \eta_{p}^{2}=.05$. A univariate ANOVA conducted on the proportion of teleological responses coded as anthropocentric indicated that Quechua participants were more anthropocentric than U.S. participants, $F(1,129)=$ 8.04, $p=.005, \eta_{\mathrm{p}}^{2}=.06$. This finding may in part reflect greater interaction with animals for subsistence purposes (such as farming) among the Quechua. For example, one adult said that a llama had a long neck "for carrying loads."

\section{Summary}

Contrary to both PT and ST, teleological explanations were not uniformly high for animals, but varied by age and cultural group. Contrary to our prediction that rates of teleology would be highest among U.S. children, Quechua children, and Quechua adults, the patterns were the reverse. These data argue against the prediction that a rural, religious, less schooled, indigenous sample would show an overall greater tendency to appeal to teleological explanations than a highly schooled, more secular sample. The data are consistent with RDT, in that teleological explanations for animals were primarily relational rather than anthropocentric. Finally, as with NLNKs, we find that effects of age are independent of those for culture.

\section{Artifacts}

Given the role of artifacts as human creations that serve intended functions, all theories would predict high rates of teleological explanations for artifact properties in all participant groups. Nonetheless, as with each of the other domains, an examination of explanations in the artifact domain permits a test of participants' overall orientation toward teleology, as well as the importance of property type. Responses are shown in Figure 1c.

There were no significant main effects of age group or country, but there was an Age Group $\times$ Country interaction, $F(1,129)=10.21$, $p=.002, \eta_{\mathrm{p}}^{2}=.07$. Quechua preschoolers provided fewer teleological explanations than the other three groups, $p s \leq .004$, Bonferroni's correction. Teleological explanations were also higher for static features than for actions, $F(1,129)=388.33, p<.001$, $\eta_{\mathrm{p}}^{2}=.75$. This difference obtained in all participant groups, but was higher in the United States and among adults, as indicated by a Property $\times$ Country interaction, $F(1,129)=5.71, p=.018, \eta_{p}^{2}=.04$, and a Property $\times$ Age Group interaction, $F(1$, 129) $=10.22, p=.002, \eta_{p}^{2}=.07$.

We also coded how often artifact teleology referred to anthropocentric functions ([clocks tick] "to give us the time") versus other functions ([chairs have four legs] "to stand well"). As predicted, anthropocentric functions were frequent, with no differences across groups (Table 2).

\section{Summary}

Teleological explanations for artifacts were high in all four groups of participants, and there were no overall effects due to age or country. Nonetheless, where there were differences, they were in the opposite direction of what would be predicted if children were more teleologically inclined overall than adults, or if Quechua participants were more teleologically inclined overall than the U.S. participants. Moreover, the stark differences due to property type (static feature vs. action) are a reminder that teleological explanations are not tied to domain per se. Instead, people flexibly generate either 
causal or teleological explanations, depending on the particular phenomena at hand.

\section{Discussion}

Is there a universal pattern of broad teleological reasoning that declines with formal schooling? We examined this question in a developmental study with participants from two cultural backgrounds: English-speaking U.S. preschoolers and adults in a middle-class, suburban, relatively secular community, and Quechua-speaking Peruvian preschoolers and adults in an indigenous, rural, more religious community. We employed a task that posed openended "why" questions ("Why do cars have wheels?") to elicit explanations coded as teleological (that pigeon has wings "so it can get away from stuff that's trying to eat them"), causal (boats float "because they're made of wood"), or nonexplanatory ("I don't know"). Items spanned three domains (NLNKs, animals, and artifacts) and two property types (parts and behaviors), to assess the extent of teleological reasoning. We organize this discussion with respect to the three issues posed in the Introduction: the domain specificity of teleological reasoning, the overall frequency of teleological reasoning, and the content of teleological reasoning in these four different groups.

The primary research question concerned teleological reasoning about NLNKs, where competing theories regarding domain specificity are most distinct. On balance, our findings support PT and run counter to the ST position that "even young children restrict purpose-based thinking to artifacts and adaptive parts of animals" (Greif et al., 2006). We found both developmental and cultural effects within the NLNK domain, as predicted by PT: higher rates of teleological explanations for children than adults, and higher rates for Quechua than U.S. participants. Although the absolute frequency of teleological explanations for NLNKs was rather low (seemingly consistent with ST), most U.S. children, Quechua children, and Quechua adults (over 70\% in each group) provided teleological as well as causal explanations for NLNKs. Thus, teleological explanations for NLNKs were more commonly endorsed when experience with formal schooling and Western scientific instruction were low.

Importantly, however, teleological reasoning was not equivalent for children and adults with less schooling. We did not obtain an interactional effect of age and culture, but rather two main effects, indicating that age and culture have independent influences. Furthermore, the underlying reasoning that participants provided, as reflected in teleological content, differed when examining the age effect versus the cultural effect: Children were more anthropocentric than adults, whereas Quechua participants were more relational than U.S. participants. Altogether these results indicate that variation in teleological reasoning associated with age is not equivalent to variation in teleological reasoning associated with culture.

Finally, although teleological reasoning was widespread among the Quechua sample, we would not wish to conclude that it was incorrectly applied. Sophisticated knowledge about ecological relations may be expressed in teleological terms (ojalehto et al., 2013), and there may be cultural differences in ontology, regarding which entities are considered living versus nonliving. Thus, rather than characterizing participants' teleological reasoning as "promiscuous" (thus implying a lack of appropriate discrimination), one might instead more precisely characterize it as broad, domain general, or unrestricted.

The second research question was whether differing rates of teleological reasoning for NLNKs, discussed earlier, were due to overarching differences in rates of adopting a teleological stance (linked to age and culture). The answer to this question was clearly "no." The distinctive features of the Quechua sample (less formal schooling, greater emphasis on religion, sensitivity to relational patterns in nature, grammaticization of -paq) did not uniformly boost teleological explanations. None of the groups were wholly teleological, as all displayed significant differences across domains, with more teleological explanations for both artifacts and animals than for NLNKs. We also found no group differences in rates of teleological explanations for artifacts. Finally, teleological reasoning went in the reverse direction for animals than NLNKs (higher for adults than children, and higher for the U.S. than Quechua participants). Again, the effects of age and culture in the animal domain did not interact, but rather were two main effects. And again, the underlying reasoning regarding animal teleology differed when examining age effects versus cultural variation-indicating that changes in teleological reasoning with age are importantly distinct from those associated with cultural variation.

The third point is that "domain" is too coarse a unit of analysis when examining teleological thought, and the nature of the property exerts powerful effects. Static features (e.g., why a bird has wings) consistently elicited more teleological expla- 
nations than action features (e.g., why a dog barks). These differences may reflect a principled distinction between static and action features - or perhaps well-chosen examples could reduce or even reverse the pattern. Regardless, the key point is that rates of teleological reasoning vary substantially depending on the property. We speculate that these differences reflect variation in the causal analysis of the features-specifically, how much a feature is seen as designed. Static features may more readily link to an item's design, given the affordances of functional parts (Kelemen, 1999a; McCarrell \& Callanan, 1995) as contrasted to actions (e.g., people do not design a vacuum cleaner to make noise; the noise is an indirect consequence of the design). For adults, features that result from intentional design are privileged for teleological explanation (Lombrozo \& Carey, 2006), and this may account for the crossage, cross-cultural finding that even controlling for domain, certain features elicit substantially more teleological explanations than others.

The underlying causal analysis of properties in the animal domain may also vary by age group and culture. U.S. adults' exposure to a Western science curriculum in which evolution is discussed with a focus on fitness and survival may promote a "design" perspective on biological features, and a corresponding teleological stance (Olson \& Labov, 2012; Ware \& Gelman, 2014). In contrast, Quechua participants were much less likely to provide teleological explanations for animal properties. Strikingly, in their nonteleological explanations for animals, Quechua children frequently mentioned contextual elements (forest, ducks, wind, bananas, etc.) that were not present in the prompt picture or question, thus arguably revealing a persistent focus on ecological relations. In contrast, Quechua adults often explained that animals have certain properties because that is just the way they are or should be (e.g., "This is how its breed is, that is why it [llama's neck] is long"), thus arguably appealing to an inevitable, natural order to the animal world. Although more research is needed, these differences suggest that causal analyses in the animal domain may reflect both developmental and cultural influences.

Another important finding is that not all types of teleological reasoning were the same. Some were anthropocentric (characterizing a property as existing for a human purpose), others indicated relational interactions among natural entities in the ecological system (e.g., a dog barks "to guide sheep"), and still others expressed a self-serving purpose (e.g., a bird has wings "to fly"). Although analyses of content do not provide a definitive test of any of the existing theories, three aspects of the data are consistent with the idea that teleological explanations may be a means of expressing ecological relations, particularly within an indigenous community, as predicted by RDT. First, teleological explanations for animals were typically relational. Second, teleological responses regarding NLNKs were more often relational for Quechua participants than for U.S. participants. Third, if we consider humans to be part of the ecology, and thus combine "relational" and "anthropocentric" into a broader relational category, the vast majority of teleological explanations of natural phenomena were relational for all groups except U.S. adults reasoning about NLNKs. At the same time, however, relational responses for animals were less frequent for Quechua participants than for U.S. participants, and there was a small but significant tendency for Quechua participants more than U.S. participants to generate anthropocentric functions for animals. We conclude that teleological explanations often express ecological relations for U.S. as well as Quechua participants, but that this does not exclude a consideration of human-centered functions for living and NLNKs.

Given that U.S. adults differed in several key respects from the other participant groups, it is important to consider factors that may contribute to these differences. We chose to study U.S. university students, given prior research specifically focused on this group (Kelemen, 1999c; Lombrozo \& Carey, 2006). All the undergraduate students in the U.S. sample had received a college preparatory education, including at minimum 3 years of Western science instruction at the high school level. In contrast, the Quechua adults received much less formal schooling (typically having attended school for at most 5 years). However, the U.S. adults in this sample also differed in other respects from the other three groups of participants, including life phase, focus on academics, and cultural practices. An important open question for future research is whether the findings from college students would extend to a broader community sample within the United States. Additionally, there are many cultural and experiential differences between the U.S. and Quechua communities (e.g., religiosity, contact with nature, agricultural practices, language differences, ritual practices regarding NLNKs such as mountains; Cummins \& Mannheim, 2011) that may interact with education to contribute to the patterns we obtained.

In conclusion, the present results indicate that cultural and developmental influences are powerful 
but independent factors in the construction of teleological reasoning. By comparing an intensively schooled, relatively secular U.S. sample that has received extensive Western scientific instruction with an indigenous Quechua-speaking community that is rural, relatively unschooled, rich in daily interactions with the natural world, steeped in religious traditions, and sensitive to relational patterns in nature, the cultural differences we obtained have multiple potential sources. Nonetheless, our findings shed light on the long-standing debate between ST and PT, offering new evidence demonstrating commonalities and variation in human cognition across cultures and development.

\section{References}

Atran, S. (1995). Causal constraints on categories. In D. Sperber, D. Premack, \& A. J. Premack (Eds.), Causal cognition: A multi-disciplinary debate (pp. 205-233). Oxford, England: Clarendon Press.

Casler, K., \& Kelemen, D. (2005). Young children's rapid learning about artifacts. Developmental Science, 8, 472480. doi:10.1111/j.1467-7687.2005.00438.x

Casler, K., \& Kelemen, D. (2008). Developmental continuity in the teleo-functional explanation: Reasoning about nature among Romanian Romani adults. Journal of Cognition and Development, 9, 340-362. doi:10.1080/ 15248370802248556

Cimpian, A., \& Markman, E. M. (2009). Information learned from generic language becomes central to children's biological concepts: Evidence from their openended explanations. Cognition, 113, 14-25. doi:10.1016/ j.cognition.2009.07.004

Cummins, T., \& Mannheim, B. (2011). The river around us, the stream within us: The traces of the Sun and Inka kinetics. RES: Anthropology and Aesthetics, 59/60, 5-21.

Cusihuamán Gutiérrez, A. (1976). Gramática quechua, Cuzco-Callao [Cuzco-Qollaw Quechua grammar]. Lima, Peru: Instituto de Estudios Peruanos.

Diesendruck, G., \& Haber, L. (2009). God's categories: The effect of religiosity on children's teleological and essentialist beliefs about categories. Cognition, 110, 100114. doi:10.1016/j.cognition.2008.11.001

Gelman, S. A., Mannheim, B., Escalante, C., \& Sánchez Tapia, I. (2015). Teleological talk in parent-child conversations in Quechua. First Language, 35, 359-376. doi:10.1080/15475441003635620

Gregory, T. R. (2009). Understanding natural selection: Essential concepts and common misconceptions. Evolution: Education and Outreach, 2, 156-175.

Greif, M. L., Kemler Nelson, D. G., Keil, F., \& Gutierrez, F. (2006). What do children want to know about animals and artifacts? Domain-specific requests for information. Psychological Science, 17, 455-459. doi:10.1111/ j.1467-9280.2006.01727.x
Henrich, J., Heine, S. J., \& Norenzayan, A. (2010). The weirdest people in the world? Behavioral and Brain Sciences, 33, 61-83. doi:10.1017/S0140525X0999152X

Keil, F. C. (1995). The growth of causal understandings of natural kinds. In D. Sperber, D. Premack, \& A. Premack (Eds.), Causal cognition: A multidisciplinary debate (pp. 234-267). New York, NY: Clarendon Press/Oxford University Press.

Kelemen, D. (1999a). Function, goals and intention: Children's teleological reasoning about objects. Trends in Cognitive Sciences, 3, 461-468. doi:10.1016/S1364-6613 (99)01402-3

Kelemen, D. (1999b). The scope of teleological thinking in preschool children. Cognition, 70, 241-272. doi:10.1016/ S0010-0277(99)00010-4

Kelemen, D. (1999c). Why are rocks pointy? Children's preference for teleological explanations of the natural world. Developmental Psychology, 35, 1440-1452. doi:10.1037/0012-1649.35.6.1440

Kelemen, D. (2012). Teleological minds: How natural intuitions about agency and purpose influence learning about evolution. In K. S., Rosengren, S. K., Brem, E. M., Evans, \& G. M., Sinatra (Eds.), Evolution challenges: Integrating research and practice in teaching and learning about evolution (pp. 66-92). Oxford: Oxford University Press.

Kelemen, D., Callanan, M. A., Casler, K., \& Pérez-Granados, D. R. (2005). Why things happen: Teleological explanation in parent-child conversations. Developmental Psychology, 41, 251-264. doi:10.1037/0012-1649.41.1.251

Kelemen, D., \& DiYanni, C. (2005). Intuitions about origins: Purpose and intelligent design in children's reasoning about nature. Journal of Cognition and Development, 6, 3-31. doi:10.1207/s15327647jcd0601_2

Kelemen, D., \& Rosset, E. (2009). The human function compunction: Teleological explanation in adults. Cognition, 111, 138-143. doi:10.1016/j.cognition.2009.01.001

Landis, J. R., \& Koch, G. G. (1977). The measurement of observer agreement for categorical data. Biometrics, 33, 159-174.

Lombrozo, T., \& Carey, S. (2006). Functional explanation and the function of explanation. Cognition, 99, 167-204. doi:10.1016/j.cognition.2004.12.009.

Lombrozo, T., Kelemen, D., \& Zaitchik, D. (2007). Inferring design: Evidence of a preference for teleological explanations in patients with Alzheimer's disease. Psychological Science, 18, 999-1006. doi:10.1111/j.1467-9280. 2007.02015.x

Mazzocchi, F. (2006). Western science and traditional knowledge: Despite their variations, different forms of knowledge can learn from each other. EMBO Reports, 7, 463-466. doi:10.1038/sj.embor.7400693

McCarrell, N. S., \& Callanan, M. A. (1995). Form-function correspondences in children's inference. Child Development, 66, 532-546. doi:10.2307/1131595.

ojalehto, B., Waxman, S. R., \& Medin, D. L. (2013). Teleological reasoning about nature: Intentional design or relational perspectives? Trends in Cognitive Sciences, 17, 166-171. doi:10.1016/j.tics.2013.02.006 
Olson, S., \& Labov, J. B. (2012). Thinking evolutionarily: Evolution education across the life sciences: Summary of a convocation. Washington, DC: National Academies Press.

Orlove, B., Chiang, J., \& Cane, M. (2002). Ethnoclimatology in the Andes: A cross-disciplinary study uncovers a scientific basis for the scheme Andean potato farmers traditionally use to predict the coming rains. American Scientist, 90, 428-435.

Ware, E. A., \& Gelman, S. A. (2014). You get what you need: An examination of purpose-based inheritance reasoning in undergraduates, preschoolers, and biological experts. Cognitive Science, 38, 197-243. doi:10.1111/cogs.12097

\section{Supporting Information}

Additional supporting information may be found in the online version of this article at the publisher's website:
Appendix S1. Description of Quechua Sample Appendix S2. Replication With Face-to-Face Interview with U.S. Adults

Appendix S3. Overview of Coding Scheme

Appendix S4. Sample Responses Coded as Teleological

Appendix S5. Coding of Causal Responses

Appendix S6. Sample Coding of Causal Responses by Explanatory Domain: Biological, Psychological, Intentional Action, Physical, and Other

Appendix S7. Mean Percentage of Causal Explanations Coded as Biological (Bio), Psychological (Psych), Intentional Action (Int. Action), Physical (Phys), or Other

Appendix S8. Individual Response Patterns

Appendix S9. Item Analyses 